\title{
NOUVELLE
}

\section{Le récepteur \\ de la ryanodine de type I}

\section{Un canal sous surveillance redox...}

Joël Lunardi, Julien Fauré, Isabelle Marty, Nicole Monnier

$>$ La contraction du muscle squelettique est causée par une brusque augmentation $\mathrm{du} \mathrm{Ca}^{2+}$ myoplasmique résultant de la vidange du réticulum sarcoplasmique (RS). Elle est déclenchée par une dépolarisation membranaire causée par l'excitation nerveuse de la fibre musculaire. La dépolarisation se propage jusqu'à des invaginations de la membrane plasmique, les tubules transverses (TT) (Figure 1). À ce niveau, la première étape du couplage excitation-contraction (CEC) est l'activation du récepteur des dihydropyridines (DHPR), un canal calcique de la membrane des $T T$ sensible au voltage.
La sortie du $\mathrm{Ca}^{2+}$ du RS, nécessaire à l'étape suivante d'activation des unités contractiles, se fait grâce à un canal calcique exprimé au niveau de la membrane du RS, le récepteur de la ryanodine de type I (RYRI). Elle est déclenchée par une interaction mécanique directe entre le DHPR activé et RYRl.

RYR1 : un complexe macromoléculaire Le relâchement de $\mathrm{Ca}^{2+}$ du RS est réalisé par un homo-tétramère de sous-unités RyRl ( $\approx 565$ kDa par sous-unité) auquel sont associées de nombreuses protéines régulatrices (FKBP12, calmoduline,

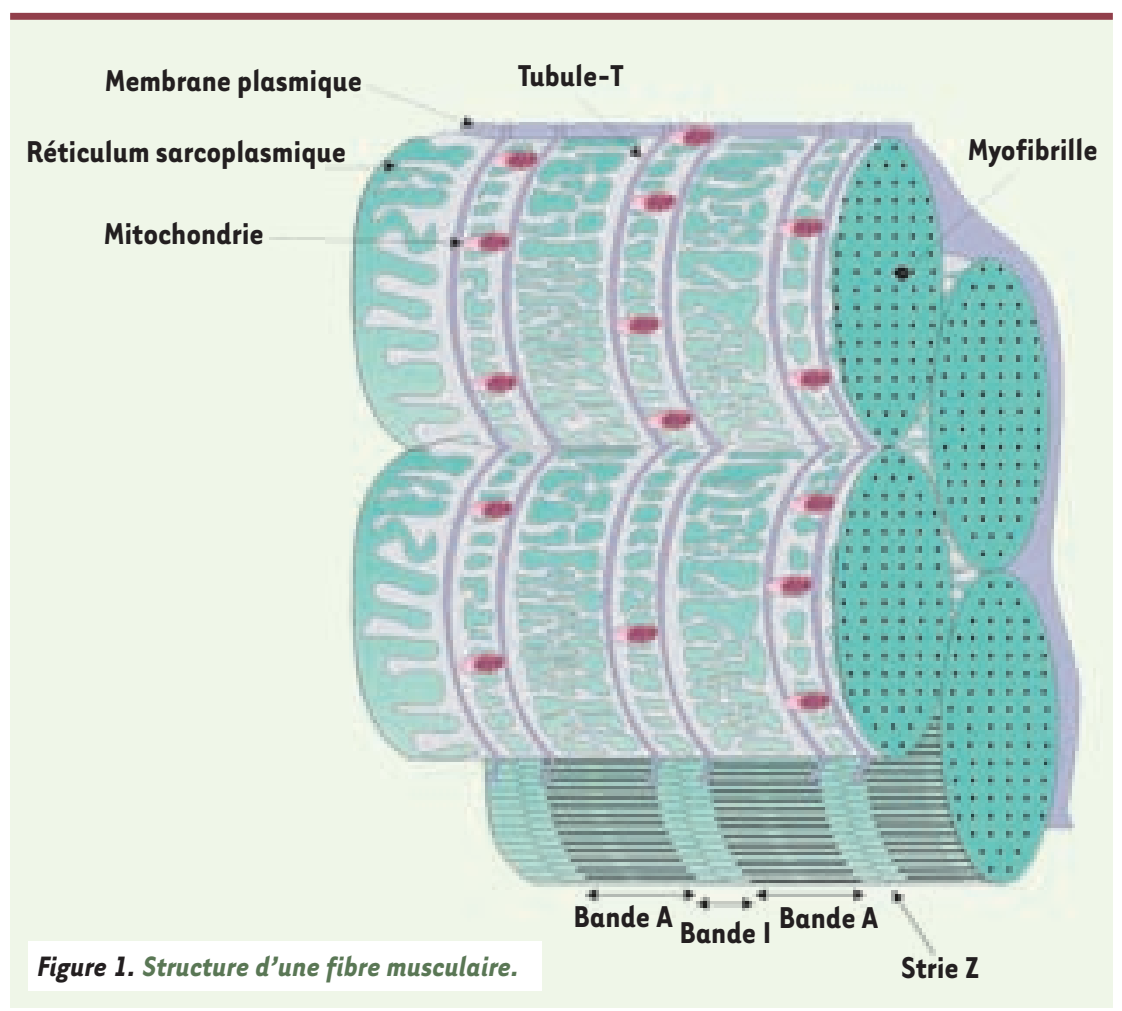

triadine, junctine, calséquestrine...). Chaque sous-unité RYRl est formée d'une partie transmembranaire carboxyterminale contenant le pore, et d'une large partie amino-terminale cytoplasmique impliquée dans la régulation de l'activité du canal. L'éventail des effecteurs endogènes de RYRl s'étend d'ions et de petites molécules $\left(\mathrm{Ca}^{2+}, \mathrm{Mg}^{2+}\right.$, adénine-nucléotides) à des polypeptides (calmoduline, FKBP12...) [1].

\section{Régulation redox de RYR 1}

RYRl est une cible pour les espèces oxygénées réactives (ROS, reactive oxygen species) et les espèces azotées réactives (RNS, reactive nitrogen species) produites lors de l'activité physique physiologique. RYRl contient un total de 100 résidus cystéinyls, la moitié se trouvant à l'état réduit dans le muscle au repos et 6-10 d'entre eux étant plus particulièrement réactifs. L'activation du canal RYRl serait modulée par des modifications redox de ces thiols par oxydation, S-nitrosylation ou alkylation. Chaque sous-unité compte entre 38 et 48 thiols à l'état libre, sensibles à l'oxydation. L'état d'activation de RYRl répond de manière biphasique à l'administration de doses croissantes d'oxydants, le maximum d'activation se situant pour un état impliquant $23 \mathrm{SH} / \mathrm{RyRl}$ [2]. L'oxyde nitrique NO est également considéré comme un modulateur physiologique de l'état redox de RYRl par S-nitrosylation [3]. Par ailleurs, l'état redox et l'activité de RYRI seraient aussi contrôlés par la pression partielle en $\mathrm{O}_{2}$ [4]. 


\section{RYR1 et physiopathologie}

\section{Un catalogue de mutations aux conséquences fonctionnelles variées}

Plus de 200 mutations du gène RYRI responsables de pathologies ont été identifiées [5]. L'hyperthermie maligne per anesthésique (HM) est une maladie pharmacogénétique dominante déclenchée par une exposition aux anesthésiques halogénés. La crise d'HM se caractérise principalement par une contracture musculaire généralisée, un emballement métabolique avec hypercapnie et une élévation très importante de la température centrale. En l'absence de traitement par un inhibiteur de RYRl, le dantrolène, l'issue de la crise est le plus souvent fatale. Les mutations HM sont principalement trouvées dans 2 domaines, $\mathrm{MH} 1$ et $\mathrm{MH} 2$, localisés dans les parties amino-terminale et centrale de RYRl.

Un second groupe de maladies associées à des mutations de RYRl sont les myopathies congénitales à cores. II s'agit d'une famille de myopathies de sévérité variable, à transmission dominante ou récessive et caractérisées par la présence d'altérations morphologiques des fibres musculaires, les cores. Les cores sont des régions de désorga- nisation sarcomérique au sein desquelles on ne trouve plus de mitochondries. Le nombre de cores, leur taille et leur localisation dans la fibre musculaire peuvent varier et cette hétérogénéité a suscité l'établissement d'une nosologie histologique de ces myopathies: central core disease (CCD), multiminicore disease $(\mathrm{MmD})$... Les mutations des formes dominantes de ces myopathies sont surtout concentrées dans la partie carboxy-terminale du canal, celles des formes récessives sont retrouvées tout au long du gène.

Les études fonctionnelles des différentes mutations du gène $R Y R I$ ont montré que certaines mutations conduisent à un canal RYRl «fuyant » entraînant une élévation du $\mathrm{Ca}^{2+}$ cytoplasmique au repos, et d'autres aboutissent à une perte du couplage fonctionnel entre le DHPR et RYRI ; un petit nombre enfin affecte la quantité de molécules RYRI présentes dans le RS.

\section{Divergence phénotypique souris/homme pour la mutation p.Y522S de RYR1}

L'équipe de Susan Hamilton a récemment créé un modèle murin knock-in pour la mutation p.y522S de RYRI [6]. Cette mutation avait été initialement

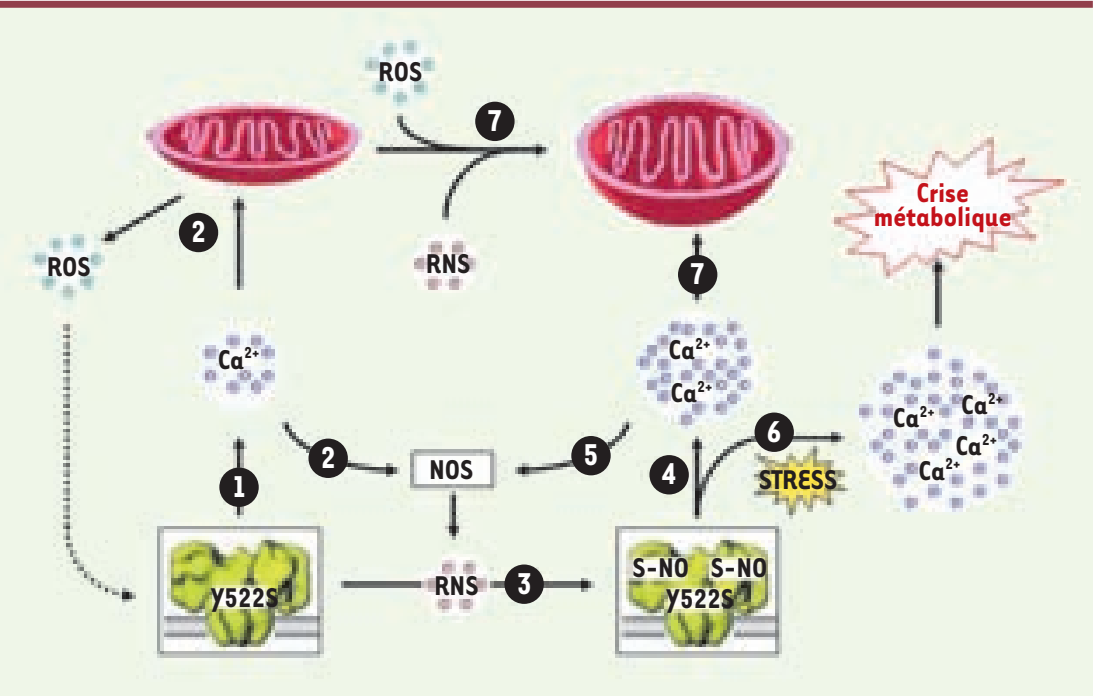

Figure 2. Modèle de dysrégulation de l'homéostasie calcique par modification redox $d u$ récepteur de la ryanodine $R Y R I$ porteur d'une mutation de type « canal fuyant ». 1. Le canal mutant s'ouvre plus facilement et entraîne une augmentation locale de la concentration en $\mathrm{Ca}^{2+}$ dans la fibre au repos. 2. Le niveau plus élevé de $\mathrm{Ca}^{2+}$ augmente la production de ROS/RNS via une action au niveau des mitochondries et des $\mathrm{NO}$ synthases (NOS). 3. Les ROS/RNS induisent la S-nitrosylation et S-glutathionylation de RYRI. 4. La S-nitrosylation augmente la sensibilité à la température de RYRl et diminue sa sensibilité à l'inhibition par le $\mathrm{Ca}^{2+}$, ce qui augmente la sortie de $\mathrm{Ca}^{2}$. 5. La sortie de $\mathrm{Ca}^{2+}$ augmente la production de ROS/RNS. 6. La sortie de $\mathrm{Ca}^{2+}$ du canal mutant est fortement augmentée en réponse à un stress (thermique, oxydatif) et conduit à un emballement métabolique avec contraction des sarcomères et activation de systèmes protéolytiques. 7. Les niveaux élevés de ROS, RNS et Ca ${ }^{2+}$ peuvent être la cause de dommages mitochondriaux chroniques. 
de la mutation p.y522S serait associée à une fuite de $\mathrm{Ca}^{2+}$ du RS responsable d'une activation de la nNOS (nitric oxide synthase) [9]. Les RNS produits entraîneraient une S-nitrosylation de la Cys 3635 de RYRl se traduisant par une augmentation de l'activation du canal muté à des températures supraphysiologiques. La fuite de $\mathrm{Ca}^{2+}$ du RS causée par la mutation p.y522S serait par ailleurs responsable d'un stress oxydatif chronique générateur d'altérations mitochondriales, elles-mêmes susceptibles d'amplifier le stress oxydatif et d'enclencher un cycle délétère stress-dommages (Figure 2). Il est cependant à noter qu'aucune altération significative des mitochondries n'a été observée dans les muscles des patients porteurs de la mutation p.y522S, ni décrite dans les muscles de nombreux autres patients porteurs de mutations de type HM.

\section{Conclusions et perspectives}

Des travaux récents réalisés chez la souris et chez l'homme montrent qu'en réponse à un exercice physique intense,
RYRl serait le siège d'un important remodelage comportant d'une part une phosphorylation de la Ser 2843 dépendante de la PKA (protein kinase A) et une S-nitrosylation des Cys 3635 et 2327 , et d'autre part une déplétion en FKBP12 [10]. La fuite importante du $\mathrm{Ca}^{2+} \mathrm{du}$ RS au travers de ces RYRl «remodelés» pourrait être la cause de dommages musculaires via une activation de la calpaïne par le $\mathrm{Ca}^{2+}$ et être l'un des composants de la limitation à l'exercice des muscles squelettiques.

Ces différentes données montrent que les modifications de l'état redox de RYRI jouent un rôle important dans la modulation de son activité canal. Les différents événements impliqués dans le remodelage de RYRl normal ou muté pourraient constituer des cibles thérapeutiques comme le suggère l'action du JTV519, une 1,4-benzothiazépine qui provoque la fixation de FKBP12 sur RYRl et qui améliorerait la fonction musculaire squelettique dans un modèle d'infarctus post-myocardique chez le rongeur [10]. $\diamond$

Ryanodine receptor:

redox state matters

\section{RÉFÉRENCES}

1. Lacampagne A, Fauconnier J, Richard S. Récepteur de la ryanodine et dysfocntionnement myocardique. Med Sci (Paris) $2007 ; 24: 399-405$.

2. Sun J, Xu L, Eu JP, et al. Classes of thiols that influence the activity of the skeletal muscle calcium release channel. J Biol Chem 2001 ; 276 : 15625-30.

3. Aghdasi, B, Reid MB, Hamilton SL. Nitric oxide protects the skeletal muscle $\mathrm{Ca}^{2+}$ release channel from oxidation induced activation. J Biol Chem $1997 ; 272: 25462-7$.

4. Eu JP, Sun J, Xu L, et al. The skeletal muscle calcium release channel: coupled $\mathrm{O}_{2}$ sensor and $\mathrm{NO}$ signaling functions. Cell 2000; $102: 499-509$.

5. Monnier N, Kozak-Ribbens G, Krivosic-Horber $R$, et al. Correlations between genotype and pharmacological, histological, functional and clinical phenotypes in malignant hyperthermia susceptibility. Hum Mutat $2005 ; 26$ : 413-25.

6. Chelu MG, Goonasekera SA, Durham WJ, et al. Heatand anesthesia-induced malignant hyperthermia in an RyRl knock-in mouse. FASEB J 2006 ; 20 : 329-30.

7. Quane KA, Keating KE Healy JMS, et al. Mutation screening of the RYRl gene in malignant hyperthermia: detection of a novel Tyr to Ser mutation in a pedigree with associated cores. Genomics $1994 ; 23$ : 236-9.

8. Monnier N, Krivosic-Horber R, Payen JF, et al. Presence of two different genetic traits in malignant hyperthermia families. Anesthesiology 2002 ; 97 : 1067-74.

9. DurhamWJ, Aracena-Parks P, Long C, et al. RyRl Snitrosylation underlies environmental heat stroke and sudden death in Y522S RyRl knockin mice. Cell 2008 ; $133:$ 53-65.

10. Bellinger AE, Reiken S, Dura M, et al. Remodeling of ryanodine receptor complex causes leaky channels: a molecular mechanism for decreased exercise capacity. Proc Natl Acad Sci USA 2008 ; 105 : 2198-2202.

\section{NOUVELle}

\section{Inflammation intestinale} et stress du réticulum endoplasmique

\section{Un lien génétique}

Eric Chevet
Avenir Inserm U889, Université Bordeaux 2, 146, rue Léo Saignat, 33076 Bordeaux, France eric.chevet@u-bordeaux2.fr

Elle facilite le repliement, l'export et la dégradation des protéines dans des conditions de stress [3]. Dans les cellules de mammifères, la réponse UPR est sous le contrôle de trois protéines transmembranaires du $R \varepsilon$, dont l'inositol requiring enzyme-l alpha and beta (IREl $\alpha \beta)$. IREl est la protéine initiatrice de la voie de signa- le rôle potentiel des voies de signalisation du stress émanant du réticulum endoplasmique (RE) dans l'inflammation et la réponse immunitaire $[1$, $2]$. Cette réponse cellulaire intégrée - appelée Unfolded Protein Response (UPR) - est activée en réponse à une accumulation de protéines mal

conformées dans la lumière du RE. Kaufman et Laurie Glimcher ont décrit

$>$ Les maladies inflammatoires de l'inintestinale à la flore microbienne. Cependant les mécanismes exacts impliqués dans ce processus inflam matoire restent mal connus. Dans deux 